\title{
Chaos Control and Bifurcation Behavior for a Sprott E System with Distributed Delay Feedback
}

\author{
Chang-Jin $\mathrm{Xu}^{1} \quad \mathrm{Yu}-\mathrm{Sen} \mathrm{Wu}^{2}$ \\ ${ }^{1}$ Guizhou Key Laboratory of Economics System Simulation, Guizhou University of Finance and Economics, Guiyang 550004, China \\ ${ }^{2}$ School of Mathematics and Statistics, Henan University of Science and Technology, Luoyang 471023, China
}

\begin{abstract}
In this paper, the problem of controlling chaos in a Sprott E system with distributed delay feedback is considered. By analyzing the associated characteristic transcendental equation, we focus on the local stability and Hopf bifurcation nature of the Sprott E system with distributed delay feedback. Some explicit formulae for determining the stability and the direction of the Hopf bifurcation periodic solutions are derived by using the normal form theory and center manifold theory. Numerical simulations for justifying the theoretical analysis are provided.
\end{abstract}

Keywords: Sprott E system, chaos control, stability, Hopf bifurcation, distributed delay.

\section{Introduction}

Since the pioneering work of Ott et al. ${ }^{[1]}$, the topics of chaos and chaotic control are growing rapidly in many different fields such as ecological system, chemical systems and biological systems, and so forth ${ }^{[2-10]}$. We all know that chaotic systems have very complicated dynamical nature, which plays an important role in many fields such as secure communications, information processing, high-performance circuit design for telecommunications ${ }^{[11]}$. Chaos, which often causes irregular behaviors in practical system, is usually undesirable. In many cases, we wish to avoid and eliminate such behaviors. Recently, many schemes such as Ott et al. ${ }^{[1]}$, feedback and non-feedback control ${ }^{[12-17]}$, observerbased control ${ }^{[12]}$, active control ${ }^{[13]}$, adaptive control ${ }^{[18,19]}$, inverse optimal control ${ }^{[20]}$, evolutionary algorithm ${ }^{[21]}$, etc., have been presented to implement the chaos control. For more related work, one can see [22-26]. In 2012, Wang and Chen ${ }^{[27]}$ reported the very surprising finding of the following new 3D autonomous chaotic Sprott E system with one stable node or stable focus

$$
\left\{\begin{array}{l}
\dot{x}=y(t) z(t)+a \\
\dot{y}=x^{2}(t)-y(t) \\
\dot{z}=1-4 x(t)
\end{array}\right.
$$

\footnotetext{
Regular paper

Manuscript received November 2, 2013; accepted March 19, 2014

This work is supported by National Natural Science Foundation of China (Nos. 11261010 and 11101126), Soft Science and Technology Program of Guizhou Province (No. 2011LKC2030), Natural Science and Technology Foundation of Guizhou Province (No. J[2012]2100), Governor Foundation of Guizhou Province (No. [2012]53) and Natural Science and Technology Foundation of Guizhou Province (2014), and Natural Science Innovation Team Project of Guizhou Province (No. [2013]14).

Recommended by Associate Editor Mohammed Chadli

(C)Institute of Automation, Chinese Academy of Science and Springer-Verlag Berlin Heidelberg 2015
}

When $a=0$, it is the Sprott E system ${ }^{[28]}$. When $a \neq 0$, the stability of the single equilibrium is fundamentally different ${ }^{[29]}$. Let $y z+a=0, x^{2}-y=0,1-4 x=0$, we can obtain that system (1) has only one stable equilibrium $E\left(x^{*}, y^{*}, z^{*}\right)=\left(\frac{1}{4}, \frac{1}{16}, 16 a\right)$ if $a>0$. Interestingly, Wang and Chen ${ }^{[29]}$ found that system (1) can generate chaotic phenomenon which is shown in Fig. 1 (Fig. 1 shows the waveform portraits and the phase portraits of system (1)).
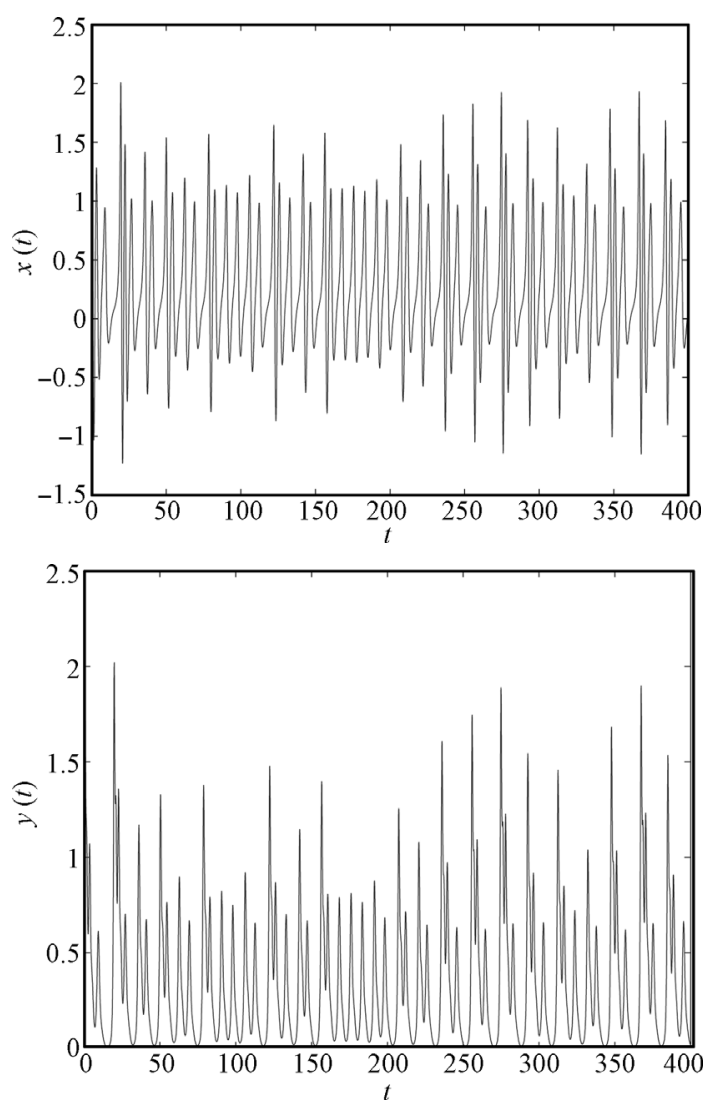

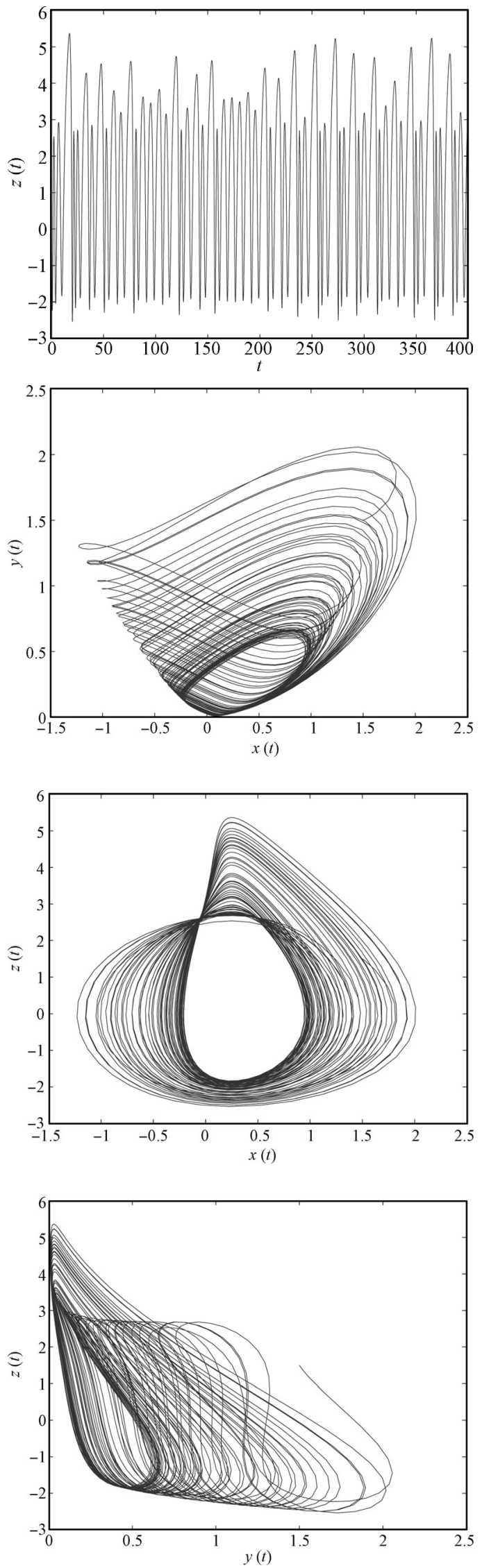
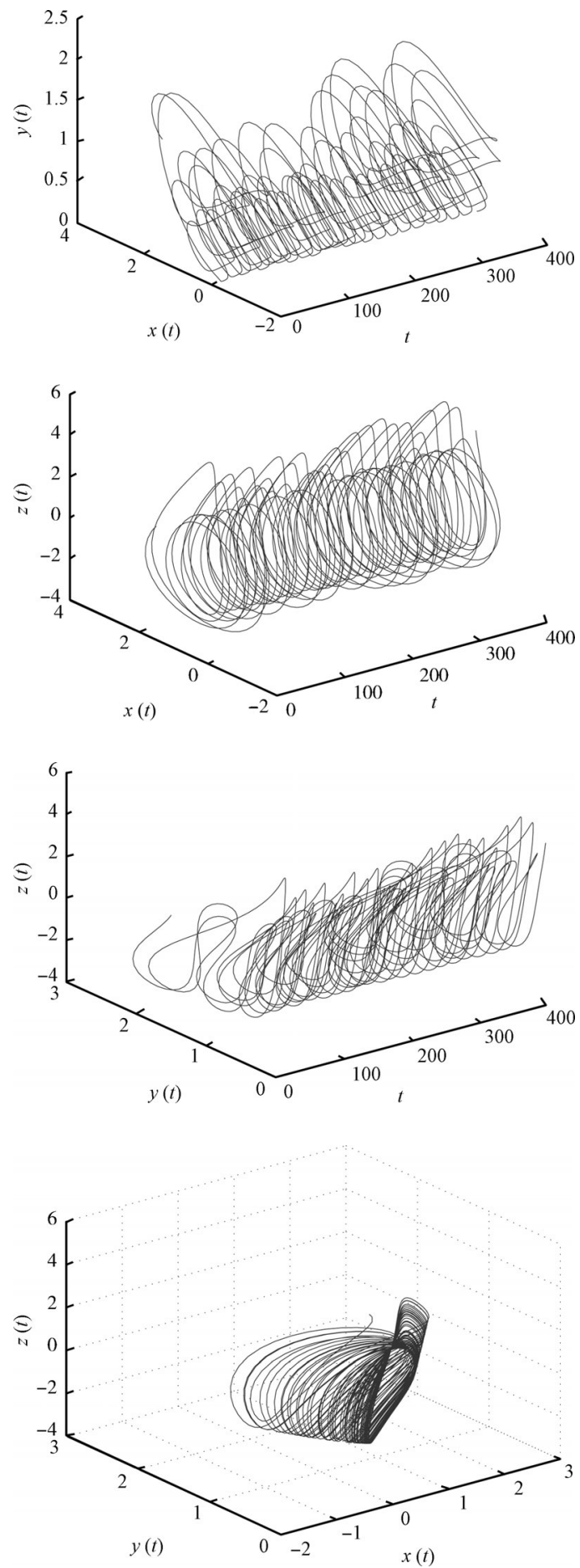

Fig. 1 Chaotic attractor of system (1) with $a=0.006$. The initial value is $(1.5,1.5,1.5)$

In this paper, we study the stability, and the local Hopf bifurcation nature for system (1). To the best of our knowledge, it is the first try to introduce continuous distributed time-delayed feedback force to control the chaos of the Sprott E system.

The remainder of the paper is organized as follows. In Section 2, we investigate the stability of the equilibrium 
and the occurrence of local Hopf bifurcations of the Sprott E system with distributed delay feedback. In Section 3, the direction and stability of the local Hopf bifurcation are established. In Section 4, numerical simulations are carried out to show the validity of chaotic control. Some main conclusions are drawn in Section 5.

\section{Stability and bifurcation analysis}

In this section, we shall study the stability of the equilibrium and the existence of local Hopf bifurcations.

In order to apply feedback control, we add a continuous distributed time delayed force

$$
b \int_{-\infty}^{0}[z(t)-z(t+s)] k(-s) \mathrm{d} s
$$

to the third equation of system (1), then system (1) takes the form

$$
\left\{\begin{array}{l}
\dot{x}=y(t) z(t)+a \\
\dot{y}=x^{2}(t)-y(t) \\
\dot{z}=1-4 x(t)+b \int_{-\infty}^{0}[z(t)-z(t+s)] k(-s) \mathrm{d} s
\end{array}\right.
$$

where $a, b>0, \int_{-\infty}^{0} k(s) \mathrm{d} s=1, \int_{-\infty}^{0} s k(s) \mathrm{d} s<+\infty$. Obviously, system (2) has the equilibrium point $E\left(x^{*}, y^{*}, z^{*}\right)=$ $\left(\frac{1}{4}, \frac{1}{16},-16 a\right)$.

Let $\bar{x}(t)=x(t)-x^{*}, \bar{y}(t)=y(t)-y^{*}, \bar{z}(t)=z(t)-z^{*}$ and still denote $\bar{x}(t), \bar{y}(t)$ and $\bar{z}(t)$ by $x(t), y(t)$ and $z(t)$, respectively, then (2) becomes

$$
\left\{\begin{array}{l}
\dot{x}=z^{*} y(t)+y^{*} z(t)+y(t) z(t) \\
\dot{y}=2 x^{*} x(t)-y(t)+x^{2}(t) \\
\dot{z}=-4 x(t)+b z(t)-b \int_{-\infty}^{0} z(t+s) k(-s) \mathrm{d} s .
\end{array}\right.
$$

The linearization of (3) near $E\left(x^{*}, y^{*}, z^{*}\right)$ is given by

$$
\left\{\begin{array}{l}
\dot{x}=z^{*} y(t)+y^{*} z(t) \\
\dot{y}=2 x^{*} x(t)-y(t) \\
\dot{z}=-4 x(t)+b z(t)-b \int_{-\infty}^{0} z(t+s) k(-s) \mathrm{d} s
\end{array}\right.
$$

whose characteristic equation appears as

$$
\begin{aligned}
& \lambda(\lambda+1)\left(\lambda-b+b \int_{-\infty}^{0} k(-s) \mathrm{e}^{\lambda s} \mathrm{~d} s\right)+4 y^{*}(\lambda+1)- \\
& 2 x^{*} z^{*}\left(\lambda-b+b \int_{-\infty}^{0} k(-s) \mathrm{e}^{\lambda s} \mathrm{~d} s\right)=0 .
\end{aligned}
$$

In this paper, we consider the weak kernel case, i.e., $k(s)=$ $\alpha \mathrm{e}^{-\alpha s}$, where $\alpha>0$. As to the general gamma kernel case, we can make a similar analysis. We give the initial condition of system (4) as

$$
\left[\begin{array}{l}
x(s) \\
y(s) \\
z(s)
\end{array}\right]=\left[\begin{array}{l}
\phi_{1}(s) \\
\phi_{2}(s) \\
\phi_{3}(s)
\end{array}\right], \quad-\infty<s \leq 0 .
$$

The characteristic equation (5) with the weak kernel case takes the form

$$
\lambda^{4}+\theta_{1}(\alpha) \lambda^{3}+\theta_{2}(\alpha) \lambda^{2}+\theta_{3}(\alpha) \lambda+\theta_{4}(\alpha)=0
$$

where

$$
\left\{\begin{array}{l}
\theta_{1}(\alpha)=\alpha-b+1 \\
\theta_{2}(\alpha)=\alpha-b-2 x^{*} z^{*}+4 y^{*} \\
\theta_{3}(\alpha)=4 y^{*}(1+\alpha)-2 x^{*} z^{*}(\alpha-b) \\
\theta_{4}(\alpha)=4 y^{*} \alpha
\end{array}\right.
$$

In view of the well known Routh-Hurwitz criterion, we can conclude that all the roots of (6) has negative real parts if the following conditions

$$
\left\{\begin{aligned}
D_{1}(\alpha)= & \theta_{1}(\alpha)=\alpha-b+1>0 \\
D_{2}(\alpha)= & \theta_{1}(\alpha) \theta_{2}(\alpha)-\theta_{3}(\alpha)= \\
& (\alpha-b+1)\left(\alpha-b-2 x^{*} z^{*}+4 y^{*}\right)- \\
& {\left[4 y^{*}(1+\alpha)-2 x^{*} z^{*}(\alpha-b)\right]>0 } \\
D_{3}(\alpha)= & \theta_{3}(\alpha) D_{2}(\alpha)-\theta_{1}^{2}(\alpha) \theta_{4}(\alpha)= \\
& {\left[4 y^{*}(1+\alpha)-2 x^{*} z^{*}(\alpha-b)\right] \times } \\
& \left\{(\alpha-b+1)\left(\alpha-b-2 x^{*} z^{*}+4 y^{*}\right)-\right. \\
& {\left.\left[4 y^{*}(1+\alpha)-2 x^{*} z^{*}(\alpha-b)\right]\right\}- } \\
& 4 y^{*} \alpha(\alpha-b+1)^{2}>0 \\
D_{4}(\alpha)= & \theta_{4}(\alpha) D_{3}(\alpha)=4 y^{*} \alpha D_{3}(\alpha)>0
\end{aligned}\right.
$$

hold true.

Based on the analysis above, we can easily obtain the following Theorem 1 .

Theorem 1. The equilibrium $E\left(x^{*}, y^{*}, z^{*}\right)$ of system (2) with the weak kernel is locally asymptotically stable if the following conditions are fulfilled:

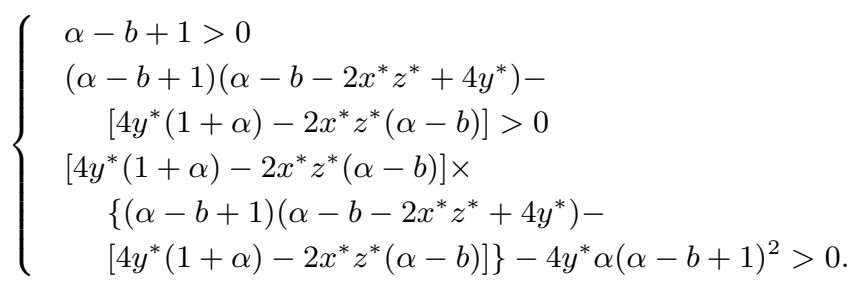

Let $\lambda_{i}(i=1,2,3,4)$ be the roots of $(6)$, then we have

$$
\left\{\begin{array}{l}
\lambda_{1}+\lambda_{2}+\lambda_{3}+\lambda_{4}=-\theta_{1}(\alpha) \\
\lambda_{1} \lambda_{2}+\lambda_{1} \lambda_{3}+\lambda_{1} \lambda_{4}+\lambda_{2} \lambda_{3}+\lambda_{2} \lambda_{4}+\lambda_{3} \lambda_{4}=\theta_{2}(\alpha) \\
\lambda_{1} \lambda_{2} \lambda_{3}+\lambda_{1} \lambda_{2} \lambda_{4}+\lambda_{1} \lambda_{3} \lambda_{4}+\lambda_{2} \lambda_{3} \lambda_{4}=-\theta_{3}(\alpha) \\
\lambda_{1} \lambda_{2} \lambda_{3} \lambda_{4}=\theta_{4}(\alpha)
\end{array}\right.
$$

If there exists an $\alpha_{0} \in \mathbf{R}^{+}$such that $D_{3}\left(\alpha_{0}\right)=0$ and $\left.\frac{\mathrm{d} D_{3}(\alpha)}{\mathrm{d} \alpha}\right|_{\alpha=\alpha_{0}} \neq 0$, then by the Routh-Hurwitz criterion, there exists a pair of purely imaginary roots, say $\lambda_{1}=$ $\bar{\lambda}_{2}=\mathrm{i} \omega_{0}(\omega \neq 0)$, and the other two roots $\lambda_{3}, \lambda_{4}$ satisfy: if $\lambda_{3}, \lambda_{4}$ are real, then $\lambda_{3}<0, \lambda_{4}<0$; if $\lambda_{3}, \lambda_{4}$ are complex conjugate, then $\operatorname{Re}\left\{\lambda_{3}\right\}=\operatorname{Re}\left\{\lambda_{4}\right\}=-\frac{\theta_{1}(\alpha)}{2}$. It is easy to calculate that

$$
\begin{aligned}
\frac{\mathrm{d}\left(\operatorname{Re}\left(\lambda_{1}\right)\right)}{\mathrm{d} \alpha}= & -\frac{\theta_{1}(\alpha)}{2\left[\theta_{1}^{3}(\alpha) \theta_{3}(\alpha)+\left(\theta_{1}(\alpha) \theta_{2}(\alpha)-2 \theta_{3}(\alpha)\right)^{2}\right]} \times \\
& \left.\frac{\mathrm{d} D_{3}(\alpha)}{\mathrm{d} \alpha}\right|_{\alpha=\alpha_{0}}
\end{aligned}
$$

thus the Hopf bifurcation occurs near $E\left(x^{*}, y^{*}, z^{*}\right)$ when $\alpha$ passes through $\alpha_{0}$. 


\section{Direction and stability of bifurcating periodic solutions}

In this section, by using techniques from normal form and center manifold theory ${ }^{[30]}$, we shall investigate the direction, stability, and period of these periodic solutions bifurcating from the equilibrium $\mathrm{E}\left(x^{*}, y^{*}, z^{*}\right)$. Let $\mu=\alpha-\alpha_{0}$, then system (3) undergoes the Hopf bifurcation at the equilibrium $E\left(x^{*}, y^{*}, z^{*}\right)$ for $\mu=0$, and then $\pm \mathrm{i} \omega_{0}$ are purely imaginary roots of the characteristic equation at the equilibrium $\mathrm{E}\left(x^{*}, y^{*}, z^{*}\right)$. System (3) can be written as an functional differential equation (FDE) in $\left.\mathbf{C}=\mathbf{C}([-\infty, 0]), \mathbf{R}^{3}\right)$ as

$$
\dot{u}(t)=A(\mu) u_{t}+R(\mu) u_{t}
$$

where $u(t)=[x(t), y(t), z(t)]^{\mathrm{T}} \in \mathbf{C}$ and $u_{t}(\theta)=u(t+\theta)=$ $[x(t+\theta), y(t+\theta), z(t+\theta)]^{\mathrm{T}} \in \mathbf{C}$, and $A$ and $R$ are given by

$$
\begin{aligned}
& A(\mu) \phi(\theta)= \\
& \qquad \begin{array}{l}
\frac{\mathrm{d} \phi(\theta)}{\mathrm{d} \theta}, \quad-\infty \leq \theta<0 \\
L \phi(\theta)+\int_{-\infty}^{0} F(s) \phi(s) \mathrm{d} s, \quad \theta=0
\end{array}
\end{aligned}
$$

and

$$
R(\mu) \phi(\theta)=\left\{\begin{array}{l}
{[0,0,0]^{\mathrm{T}}, \quad-\infty \leq \theta<0} \\
{\left[f_{1}, f_{2}, 0\right]^{\mathrm{T}}, \quad \theta=0}
\end{array}\right.
$$

respectively, where $\phi(\theta)=\left[\phi_{1}(\theta), \phi_{2}(\theta), \phi_{3}(\theta)\right]^{\mathrm{T}} \in \mathbf{C}$ and $f_{1}=\phi_{2}(0) \phi_{3}(0), f_{2}=\phi_{1}^{2}(0)$.

For $\psi \in \mathbf{C}\left([0,+\infty],\left(\mathbf{R}^{3}\right)^{*}\right)$, define

$$
A^{*} \psi(s)=\left\{\begin{array}{l}
-\frac{\mathrm{d} \psi(s)}{\mathrm{d} s}, \quad s \in(0,+\infty] \\
L^{\mathrm{T}} \psi(0)+\int_{-\infty}^{0} F^{\mathrm{T}}(t) \psi(-t) \mathrm{d} s, \quad s=0 .
\end{array}\right.
$$

For $\phi \in \mathbf{C}\left([-\infty, 0], \mathbf{R}^{3}\right)$ and $\psi \in \mathbf{C}\left([0,+\infty],\left(\mathbf{R}^{3}\right)^{*}\right)$, define the bilinear form

$$
\langle\psi, \phi\rangle=\bar{\psi}(0) \phi(0)-\int_{-\infty}^{0} \int_{\xi=0}^{\theta} \bar{\psi}(\xi-\theta) \mathrm{d} \eta(\theta) \phi(\xi) \mathrm{d} \xi
$$

where $\eta(\theta)=\eta(\theta, 0)$, the $A=A(0)$ and $A^{*}$ are adjoint operators. By the discussions in Section 2, we know that $\pm \mathrm{i} \omega_{0}$ are eigenvalues of $A(0)$, and they are also eigenvalues of $A^{*}$ corresponding to $i \omega_{0}$ and $-\mathrm{i} \omega_{0}$, respectively. Assume that $q(\theta)=\left(1, a_{1}, a_{2}\right)^{\mathrm{T}} \mathrm{e}^{\mathrm{i} \omega_{0} \theta}$ is the eigenvector of $A(0)$ corresponding to $\mathrm{i} \omega_{0}$, then we have $A(0) q(0)=\mathrm{i} \omega_{0} q(0)$, i.e.,

$$
\begin{aligned}
L q(0)+ & \int_{-\infty}^{0} F(s) q(s) \mathrm{d} s=\left[\begin{array}{ccc}
0 & z^{*} & y^{*} \\
2 x^{*} & -1 & 0 \\
-4 & 0 & b
\end{array}\right]\left[\begin{array}{c}
1 \\
a_{1} \\
a_{2}
\end{array}\right]+ \\
& \int_{-\infty}^{0}\left[\begin{array}{ccc}
0 & 0 & 0 \\
0 & 0 & 0 \\
0 & 0 & b k(-s)
\end{array}\right]\left[\begin{array}{c}
1 \\
a_{1} \\
a_{2}
\end{array}\right] \mathrm{e}^{\mathrm{i} \omega_{0} s} \mathrm{~d} s= \\
& {\left[\begin{array}{c}
a_{1} z^{*}+a_{2} y^{*} \\
2 x^{*}-a_{1} \\
-4+b a_{2}-b a_{2} \chi^{(1)}
\end{array}\right]=\left[\begin{array}{c}
\mathrm{i} \omega_{0} \\
\mathrm{i} \omega_{0} a_{1} \\
\mathrm{i} \omega_{0} a_{2}
\end{array}\right] }
\end{aligned}
$$

where

$$
\chi^{(1)}=\int_{-\infty}^{0} k(-s) \mathrm{e}^{\mathrm{i} \omega_{0} s} \mathrm{~d} s=\frac{\alpha}{\alpha+\mathrm{i} \omega_{0}} .
$$

We can obtain

$$
q(\theta)=\left[1, a_{1}, a_{2}\right]^{\mathrm{T}} \mathrm{e}^{\mathrm{i} \omega_{0} \theta}
$$

where

$$
a_{1}=\frac{2 x^{*}}{\mathrm{i} \omega_{0}+1}, \quad a_{2}=\frac{4}{b-b \chi^{(1)}-\mathrm{i} \omega_{0}} .
$$

Assume that $q^{*}(s)=D\left[1, a_{1}^{*}, a_{2}^{*}\right]^{\mathrm{T}} \mathrm{e}^{\mathrm{i} \omega_{0} s}(0 \leq s<+\infty)$ is the eigenvector of $A^{*}(0)$ corresponding to $-\mathrm{i} \omega_{0}$, then we have $A^{*}(0) q^{*}(0)=\mathrm{i} \omega_{0} q^{*}(0)$, i.e.,

$$
\begin{aligned}
L^{\mathrm{T}} q^{*}(0)+\int_{-\infty}^{0} F^{*}(s) q^{*}(-s) \mathrm{d} s= \\
\quad\left[\begin{array}{ccc}
0 & 2 x^{*} & -4 \\
z^{*} & -1 & 0 \\
y^{*} & 0 & b
\end{array}\right]\left[\begin{array}{c}
D \\
D a_{1}^{*} \\
D a_{2}^{*}
\end{array}\right]+ \\
\quad \int_{-\infty}^{0}\left[\begin{array}{ccc}
0 & 0 & 0 \\
0 & 0 & 0 \\
0 & 0 & b k(-s)
\end{array}\right]\left[\begin{array}{c}
D \\
D a_{1}^{*} \\
D a_{2}^{*}
\end{array}\right] \mathrm{e}^{-\mathrm{i} \omega_{0} s} \mathrm{~d} s= \\
{\left[\begin{array}{c}
2 x^{*} a_{1}^{*}-4 a_{2}^{*} \\
z^{*}-a_{1}^{*} \\
y^{*}+b a_{2}^{*}-b \chi^{(2)}
\end{array}\right]=\left[\begin{array}{c}
-\mathrm{i} \omega_{0} D \\
-\mathrm{i} \omega_{0} a_{1}^{*} D \\
-\mathrm{i} \omega_{0} a_{2}^{*} D
\end{array}\right] }
\end{aligned}
$$

where

$$
\chi^{(2)}=\int_{-\infty}^{0} k(-s) \mathrm{e}^{-\mathrm{i} \omega_{0} s} \mathrm{~d} s=\frac{\alpha}{\alpha-\mathrm{i} \omega_{0}} .
$$

We can obtain

$$
q^{*}(s)=D\left[1, a_{1}^{*}, a_{2}^{*}\right]^{\mathrm{T}} \mathrm{e}^{\mathrm{i} \omega_{0} s}
$$

where

$$
a_{1}^{*}=\frac{z^{*}}{1-\mathrm{i} \omega_{0}}, \quad a_{2}^{*}=\frac{b \chi^{(2)}-y^{*}}{b+\mathrm{i} \omega_{0}} .
$$

If we choose

$$
D=\frac{1}{1+a_{1}^{*} \bar{a}_{1}+a_{2}^{*} \bar{a}_{2}+b a_{2}^{*} \bar{a}_{2} \int_{-\infty}^{0} \theta \mathrm{e}^{-\mathrm{i} \omega_{0} \theta} k(-\theta) \mathrm{d} \theta}
$$

then $<q^{*}(s), q(\theta) \geq 1$ and $<q^{*}(s), \bar{q}(\theta) \geq 0$.

Next, we use the same notations as those in Hassard ${ }^{[30]}$ and we first compute the coordinates to describe the center manifold $C_{0}$ at $\mu=0$. Let $u_{t}$ be the solution of (11) when $\mu=0$.

Define

$$
z(t)=\left\langle q^{*}, u_{t}\right\rangle W(t, \theta)=u_{t}(\theta)-2 \operatorname{Re}\{z(t) q(\theta)\}
$$

on the center manifold $C_{0}$, and we have

$$
W(t, \theta)=W(z(t), \bar{z}(t), \theta)
$$

where

$W(z(t), \bar{z}(t), \theta)=W(z, \bar{z})=W_{20} \frac{z^{2}}{2}+W_{11} z \bar{z}+W_{02} \frac{\bar{z}^{2}}{2}+\cdots$

and $z$ and $\bar{z}$ are local coordinates for center manifold $C_{0}$ in the direction of $q^{*}$ and $\bar{q}^{*}$. Noting that $W$ is also real 
if $u_{t}$ is real, we consider only real solutions. For solutions $u_{t} \in C_{0}$ of (11),

$$
\begin{aligned}
\dot{z}(t)= & \mathrm{i} \omega_{0} z+\bar{q}^{*}(\theta) f[0, W(z, \bar{z}, \theta)]+2 \operatorname{Re}\{z q(\theta)\} \stackrel{\text { def }}{=} \\
& \mathrm{i} \omega_{0} z+\bar{q}^{*}(0)\left[f_{1}, f_{2}, 0\right]^{\mathrm{T}} .
\end{aligned}
$$

That is

$$
\dot{z}(t)=\mathrm{i} \omega_{0} z+g(z, \bar{z})
$$

where

$$
g(z, \bar{z})=g_{20} \frac{z^{2}}{2}+g_{11} z \bar{z}+g_{02} \frac{\bar{z}^{2}}{2}+\cdots .
$$

Let $f_{0}=\left[f_{1}, f_{2}\right]^{\mathrm{T}}$. Hence, we have

$$
\begin{aligned}
g(z, \bar{z})= & \bar{q}^{*}(0) f_{0}(z, \bar{z})=f\left(0, u_{t}\right)= \\
& \bar{D}\left(a_{1} a_{2}+\bar{a}_{1}^{*}\right) z^{2}+\bar{D}\left(2 \operatorname{Re}\left\{a_{1} \bar{a}_{2}\right\}+2 \bar{a}_{1}^{*}\right) z \bar{z}+ \\
& \bar{D}\left(\bar{a}_{1} \bar{a}_{2}+\bar{a}_{1}^{*}\right) \bar{z}^{2}+\bar{D}\left[\frac{1}{2} \bar{a}_{1} W_{20}^{(3)}(0)+\right. \\
& \frac{1}{2} \bar{a}_{2} W_{20}^{(3)}(0)+\bar{a}_{1} W_{11}^{(3)}(0)+\bar{a}_{2} W_{11}^{(2)}(0)+ \\
& \left.\bar{a}_{1}^{*}\left(W_{20}^{(1)}(0)+2 W_{11}^{(1)}(0)\right)\right] z^{2} \bar{z}+\text { high order term } .
\end{aligned}
$$

Then, we get

$$
\begin{aligned}
g_{20}= & 2 \bar{D}\left(a_{1} a_{2}+\bar{a}_{1}^{*}\right) \\
g_{11}= & 2 \bar{D}\left(\operatorname{Re}\left\{a_{1} \bar{a}_{2}\right\}+\bar{a}_{1}^{*}\right) \\
g_{02}= & 2 \bar{D}\left(\bar{a}_{1} \bar{a}_{2}+\bar{a}_{1}^{*}\right) \\
g_{21}= & 2 \bar{D}\left[\frac{1}{2} \bar{a}_{1} W_{20}^{(3)}(0)+\frac{1}{2} \bar{a}_{2} W_{20}^{(3)}(0)+\right. \\
& \bar{a}_{1} W_{11}^{(3)}(0)+\bar{a}_{2} W_{11}^{(2)}(0)+ \\
& \left.\bar{a}_{1}^{*}\left(W_{20}^{(1)}(0)+2 W_{11}^{(1)}(0)\right)\right] .
\end{aligned}
$$

Since there exist unknowns $W_{20}^{(1)}(0), \quad W_{20}^{(3)}(0)$, $W_{11}^{(1)}(0), W_{11}^{(2)}(0), W_{11}^{(3)}(0)$ in $g_{21}$, we still need to compute them.

It follows from (11) and (14) that

$W^{\prime}=\left\{\begin{array}{l}A W-2 \operatorname{Re}\left\{\bar{q}^{*}(0)\left[f_{1}, f_{2}, 0\right]^{\mathrm{T}} q(\theta)\right\},-\infty \leq \theta<0 \\ A W-2 \operatorname{Re}\left\{\bar{q}^{*}(0)\left[f_{1}, f_{2}, 0\right]^{\mathrm{T}} q(\theta)\right\}+\left[f_{1}, f_{2}, 0\right]^{\mathrm{T}}, \\ \theta=0\end{array}\right.$

$$
\stackrel{\text { def }}{=} A W+H(z, \bar{z}, \theta)
$$

where

$$
H(z, \bar{z}, \theta)=H_{20}(\theta) \frac{z^{2}}{2}+H_{11}(\theta) z \bar{z}+H_{02}(\theta) \frac{\bar{z}^{2}}{2}+\cdots
$$

Comparing the coefficients, we obtain

$$
\begin{gathered}
\left(A W-2 \mathrm{i} \omega_{0}\right) W_{20}=-H_{20}(\theta) \\
A W_{11}(\theta)=-H_{11}(\theta) .
\end{gathered}
$$

For $\theta \in[-\infty, 0)$,

$$
\begin{aligned}
H(z, \bar{z}, \theta)= & -\bar{q}^{*}(0) f_{0} q(\theta)-q^{*}(0) \overline{f_{0}} \bar{q}(\theta)= \\
& -g(z, \bar{z}) q(\theta)-\bar{g}(z, \bar{z}) \bar{q}(\theta) .
\end{aligned}
$$

Comparing the coefficients of (21) with (18) gives that

$$
\begin{aligned}
& H_{20}(\theta)=-g_{20} q(\theta)-\bar{g}_{02} \bar{q}(\theta) \\
& H_{11}(\theta)=-g_{11} q(\theta)-\bar{g}_{11} \bar{q}(\theta) .
\end{aligned}
$$

From (19), (22) and the definition of $A$, we get

$$
\dot{W}_{20}(\theta)=2 \mathrm{i} \omega_{0} W_{20}(\theta)+g_{20} q(\theta)+g_{02}^{-} \bar{q}(\theta) .
$$

Noting that $q(\theta)=q(0) \mathrm{e}^{\mathrm{i} \omega_{0} \theta}$, we have

$$
W_{20}(\theta)=\frac{\mathrm{i} g_{20}}{\omega_{0}} q(0) \mathrm{e}^{\mathrm{i} \omega_{0} \theta}+\frac{\mathrm{i} \bar{g}_{02}}{3 \omega_{0}} \bar{q}(0) \mathrm{e}^{-\mathrm{i} \omega_{0} \theta}+E_{1} \mathrm{e}^{2 \mathrm{i} \omega_{0} \theta}
$$

where $E_{1}=\left[E_{1}^{(1)}, E_{1}^{(2)}, E_{1}^{(3)}\right] \in \mathbf{R}^{3}$ is a constant vector. Similarly, from $(20),(23)$ and the definition of $A$, we have

$$
\begin{gathered}
\dot{W}_{11}(\theta)=g_{11} q(\theta)+g_{11}^{-} \bar{q}(\theta) \\
W_{11}(\theta)=-\frac{\mathrm{i} g_{11}}{\omega_{0}} q(0) \mathrm{e}^{\mathrm{i} \omega_{0} \theta}+\frac{\mathrm{i} \bar{g}_{11}}{\omega_{0}} \bar{q}(0) \mathrm{e}^{-\mathrm{i} \omega_{0} \theta}+E_{2}
\end{gathered}
$$

where $E_{2}=\left[E_{2}^{(1)}, E_{2}^{(2)}, E_{2}^{(3)}\right] \in \mathbf{R}^{3}$ is a constant vector.

In what follows, we shall seek appropriate $E_{1}, E_{2}$ in (25), (27), respectively. It follows from the definition of $A$ and (22), (23) that

$$
\int_{-1}^{0} \mathrm{~d} \eta(\theta) W_{20}(\theta)=2 \mathrm{i} \omega_{0} W_{20}(0)-H_{20}(0)
$$

and

$$
\int_{-1}^{0} \mathrm{~d} \eta(\theta) W_{11}(\theta)=-H_{11}(0)
$$

where $\eta(\theta)=\eta(0, \theta)$.

From (22) and (23), we have

$$
H_{20}(0)=-g_{20} q(0)-\bar{g}_{02} \bar{q}(0)+2\left[\begin{array}{c}
a_{1} a_{2} \\
1 \\
0
\end{array}\right]
$$

$$
H_{11}(0)=-g_{11} q(0)-\bar{g}_{11}(0) \bar{q}(0)+2\left[\begin{array}{c}
\operatorname{Re}\left\{a_{1} \bar{a}_{2}\right\} \\
1 \\
0
\end{array}\right] \text {. }
$$

Noting that

$$
\begin{gathered}
\left(\mathrm{i} \omega_{0} I-\int_{-1}^{0} \mathrm{e}^{\mathrm{i} \omega_{0} \theta} \mathrm{d} \eta(\theta)\right) q(0)=0 \\
\left(-\mathrm{i} \omega_{0} I-\int_{-1}^{0} \mathrm{e}^{-\mathrm{i} \omega_{0} \theta} \mathrm{d} \eta(\theta)\right) \bar{q}(0)=0
\end{gathered}
$$

and substituting (25) and (30) into (28), we have

$$
\left(2 \mathrm{i} \omega_{0} I-\int_{-1}^{0} \mathrm{e}^{2 \mathrm{i} \omega_{0} \theta} \mathrm{d} \eta(\theta)\right) E_{1}=2\left[\begin{array}{c}
a_{1} a_{2} \\
1 \\
0
\end{array}\right] .
$$


That is

$$
\left[\begin{array}{ccc}
2 \mathrm{i} \omega_{0} & -z^{*} & -y^{*} \\
2 x^{*} & 2 \mathrm{i} \omega_{0}+1 & 0 \\
4 & 0 & 2 \mathrm{i} \omega_{0}-b+b \chi^{(3)}
\end{array}\right] E_{1}=2\left[\begin{array}{c}
a_{1} a_{2} \\
1 \\
0
\end{array}\right]
$$

where

$$
\chi^{(3)}=\int_{-\infty}^{0} k(-s) \mathrm{e}^{2 \mathrm{i} \omega_{0} s} \mathrm{~d} s=\frac{\alpha}{\alpha+2 \mathrm{i} \omega_{0}} .
$$

It follows that

$$
E_{1}^{(1)}=\frac{\Delta_{11}}{\Delta_{1}}, E_{1}^{(2)}=\frac{\Delta_{12}}{\Delta_{1}}, E_{1}^{(3)}=\frac{\Delta_{13}}{\Delta_{1}}
$$

where

$$
\begin{aligned}
& \Delta_{1}=\operatorname{det}\left[\begin{array}{ccc}
2 \mathrm{i} \omega_{0} & -z^{*} & -y^{*} \\
2 x^{*} & 2 \mathrm{i} \omega_{0}+1 & 0 \\
4 & 0 & 2 \mathrm{i} \omega_{0}-b+b \chi^{(3)}
\end{array}\right] \\
& \Delta_{11}=2 \operatorname{det}\left[\begin{array}{ccc}
a_{1} a_{2} & -z^{*} & -y^{*} \\
1 & 2 \mathrm{i} \omega_{0}+1 & 0 \\
0 & 0 & 2 \mathrm{i} \omega_{0}-b+b \chi^{(3)}
\end{array}\right] \\
& \Delta_{12}=2 \operatorname{det}\left[\begin{array}{ccc}
2 \mathrm{i} \omega_{0} & a_{1} a_{2} & -y^{*} \\
2 x^{*} & 1 & 0 \\
4 & 0 & 2 \mathrm{i} \omega_{0}-b+b \chi^{(3)}
\end{array}\right] \\
& \Delta_{13}=2 \operatorname{det}\left[\begin{array}{ccc}
2 \mathrm{i} \omega_{0} & -z^{*} & a_{1} a_{2} \\
2 x^{*} & 2 \mathrm{i} \omega_{0}+1 & 1 \\
4 & 0 & 0
\end{array}\right] .
\end{aligned}
$$

Similarly, substituting (26) and (31) into (29), we have

$$
\left(\int_{-1}^{0} \mathrm{~d} \eta(\theta)\right) E_{2}=2\left[\begin{array}{c}
\operatorname{Re}\left\{a_{1} \bar{a}_{2}\right\} \\
1 \\
0
\end{array}\right]
$$

That is

$$
\left[\begin{array}{ccc}
0 & z^{*} & y^{*} \\
2 x^{*} & -1 & 0 \\
-4 & 0 & b+b \chi^{(1)}
\end{array}\right] E_{2}=2\left[-\operatorname{Re}\left\{a_{1} \bar{a}_{2}\right\},-1,0\right]^{\mathrm{T}}
$$

It follows that

$$
E_{2}^{(1)}=\frac{\Delta_{21}}{\Delta_{2}}, E_{2}^{(2)}=\frac{\Delta_{22}}{\Delta_{2}}, E_{2}^{(3)}=\frac{\Delta_{23}}{\Delta_{2}}
$$

where

$$
\begin{aligned}
& \Delta_{2}=\operatorname{det}\left[\begin{array}{ccc}
0 & z^{*} & y^{*} \\
2 x^{*} & -1 & 0 \\
-4 & 0 & b+b \chi^{(1)}
\end{array}\right] \\
& \Delta_{21}=2 \operatorname{det}\left[\begin{array}{ccc}
-\operatorname{Re}\left\{a_{1} \bar{a}_{2}\right\} & z^{*} & y^{*} \\
-1 & -1 & 0 \\
0 & 0 & b+b \chi^{(1)}
\end{array}\right] \\
& \Delta_{22}=2 \operatorname{det}\left[\begin{array}{ccc}
0 & -\operatorname{Re}\left\{a_{1} \bar{a}_{2}\right\} & y^{*} \\
2 x^{*} & -1 & 0 \\
-4 & 0 & b+b \chi^{(1)}
\end{array}\right]
\end{aligned}
$$

$$
\Delta_{23}=2 \operatorname{det}\left[\begin{array}{ccc}
0 & z^{*} & -\operatorname{Re}\left\{a_{1} \bar{a}_{2}\right\} \\
2 x^{*} & -1 & -1 \\
-4 & 0 & 0
\end{array}\right] \text {. }
$$

From (25), (27), (32) and (33), we can calculate $g_{21}$ and derive the following values:

$$
\left\{\begin{array}{l}
c_{1}(0)=\frac{\mathrm{i}}{2 \omega_{0}}\left(g_{20} g_{11}-2\left|g_{11}\right|^{2}-\frac{\left|g_{02}\right|^{2}}{3}\right)+\frac{g_{21}}{2} \\
\mu_{2}=-\frac{\operatorname{Re}\left\{c_{1}(0)\right\}}{\operatorname{Re}\left\{\lambda^{\prime}\left(\alpha_{0}\right)\right\}} \\
\beta_{2}=2 \operatorname{Re}\left(c_{1}(0)\right) \\
T_{2}=-\frac{\operatorname{Im}\left\{c_{1}(0)\right\}+\mu_{2} \operatorname{Im}\left\{\lambda^{\prime}\left(\alpha_{0}\right)\right\}}{\omega_{0}}
\end{array}\right.
$$

which determine the quantities of bifurcating periodic solutions on the center manifold $C_{0}$ at the critical value $\alpha_{0}$, namely, we have the following result.

Theorem 2. The periodic solution is supercritical (subcritical) if $\mu_{2}>0\left(\mu_{2}<0\right)$; the bifurcating periodic solutions are orbitally asymptotically stable with asymptotical phase (unstable) if $\beta_{2}<0\left(\beta_{2}>0\right)$; the periods of the bifurcating periodic solutions increase (decrease) if $T_{2}>0$ $\left(T_{2}<0\right)$.

\section{Computer simulations}

In this section, we present some numerical results of system (2) to verify the analytical predictions obtained in the previous section. From Section 3, we may determine the direction of a Hopf bifurcation and the stability of the bifurcation periodic solutions. Let us consider the following system:

$$
\left\{\begin{array}{l}
\dot{x}=y(t) z(t)+0.006 \\
\dot{y}=x^{2}(t)-y(t) \\
\dot{z}=1-4 x(t)+0.5 \int_{-\infty}^{0}(z(t)-z(t+s)) k(-s) \mathrm{d} s
\end{array}\right.
$$

where $k(s)=\alpha \mathrm{e}^{-\alpha s}, \alpha>0$. Let $y z+0.006=0, x^{2}-y=$ $0,1-4 x=0$. It is easy to see that system (35) has an equilibrium $\mathrm{E}\left(\frac{1}{4}, \frac{1}{16}, \frac{1}{4},-\frac{12}{125}\right)$ and all the conditions indi- 
cated in Theorem 2 are satisfied. When $\tau=0$, the equilibrium $\mathrm{E}\left(\frac{1}{4}, \frac{1}{16}, \frac{1}{4},-\frac{12}{125}\right)$ is asymptotically stable. By means of Matlab 7.0, we get $\alpha_{0} \approx 0.4512$. Thus the equilibrium $\mathrm{E}\left(\frac{1}{4}, \frac{1}{16}, \frac{1}{4},-\frac{12}{125}\right)$ is stable when $\alpha<\alpha_{0}$ which is illustrated by the computer simulations (see Fig. 2). When $\alpha$ passes through the critical value $\alpha_{0}$, the equilibrium $\mathrm{E}\left(\frac{1}{4}, \frac{1}{16}, \frac{1}{4},-\frac{12}{125}\right)$ loses its stability and a Hopf bifurcation occurs, i.e., a family of periodic solutions bifurcations from the equilibrium $\mathrm{E}\left(\frac{1}{4}, \frac{1}{16}, \frac{1}{4},-\frac{12}{125}\right)$. It follows from the formulae (34) presented in Section 3 that $\mu_{2}>0$ and $\beta_{2}<0$. Then the direction of the Hopf bifurcation is $\alpha>\alpha_{0}$, and these bifurcating periodic solutions from $\mathrm{E}\left(\frac{1}{4}, \frac{1}{16}, \frac{1}{4},-\frac{12}{125}\right)$ around $\alpha_{0}$ are stable, which are depicted in Fig. 3.
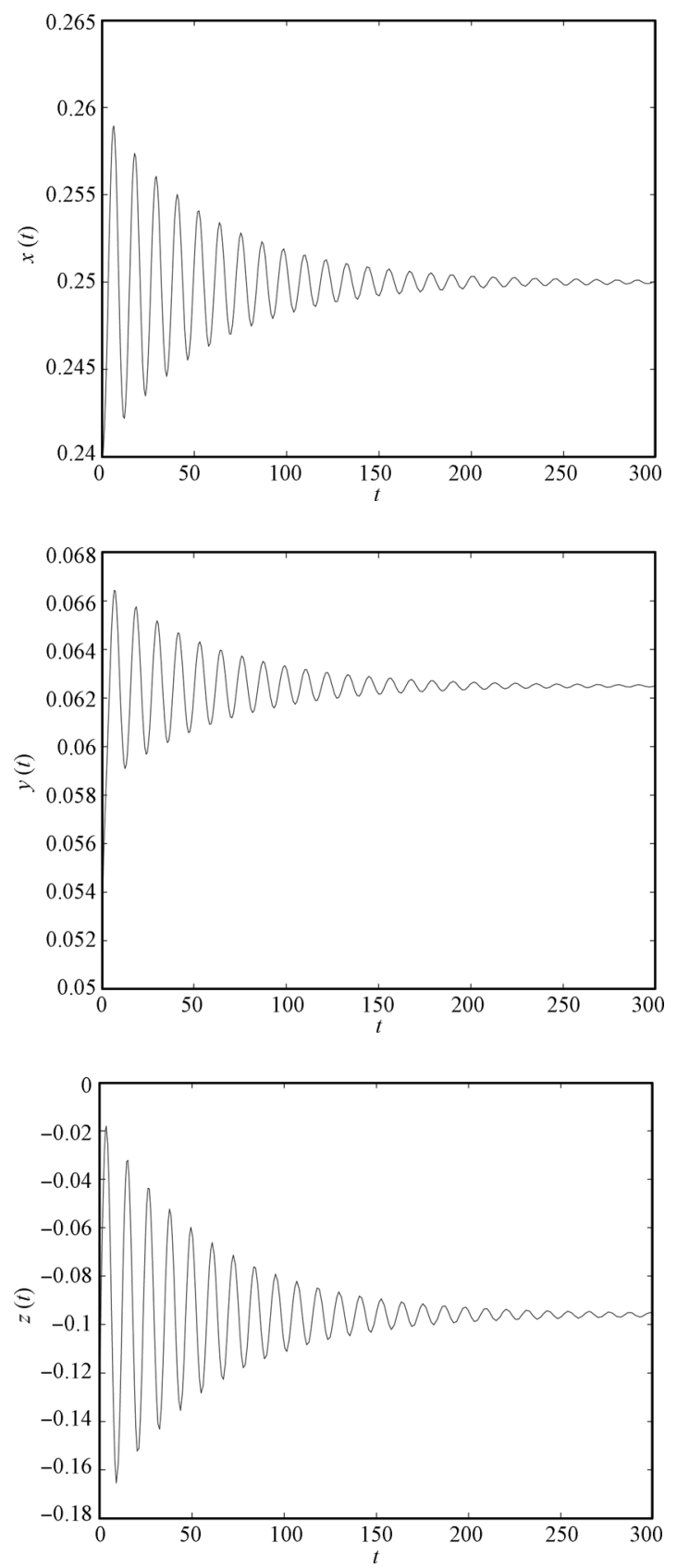
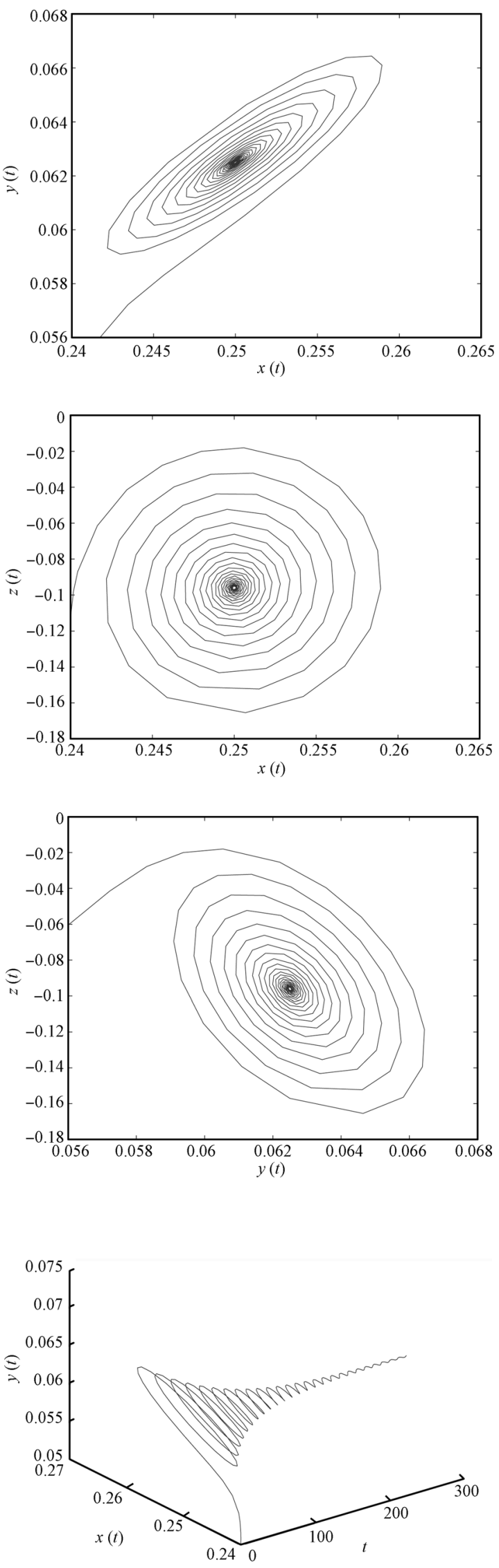


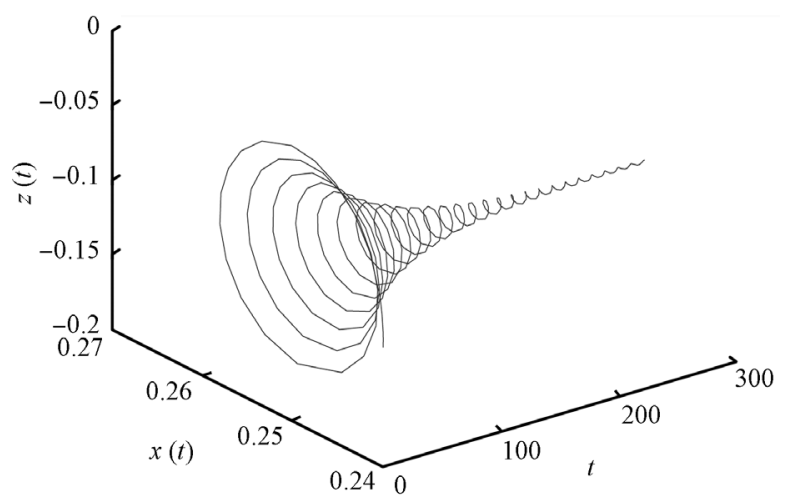

conditions are fulfilled, then the Sprott E system is asymptotically stable for $\alpha<\alpha_{0}$ and when $\alpha$ passes through $\alpha_{0}$, a sequence of Hopf bifurcations occur around the equilibrium $\mathrm{E}\left(x^{*}, y^{*}, z^{*}\right)$, namely, a family of periodic orbits bifurcate from the the equilibrium $\mathrm{E}\left(x^{*}, y^{*}, z^{*}\right)$, which implies the chaos of this system can be suppress. Some numerical simulations are included to visualize the theoretical findings. Moreover, the control method used in this paper can be applicable to other chaotic systems. We will carry our some related work in near future.
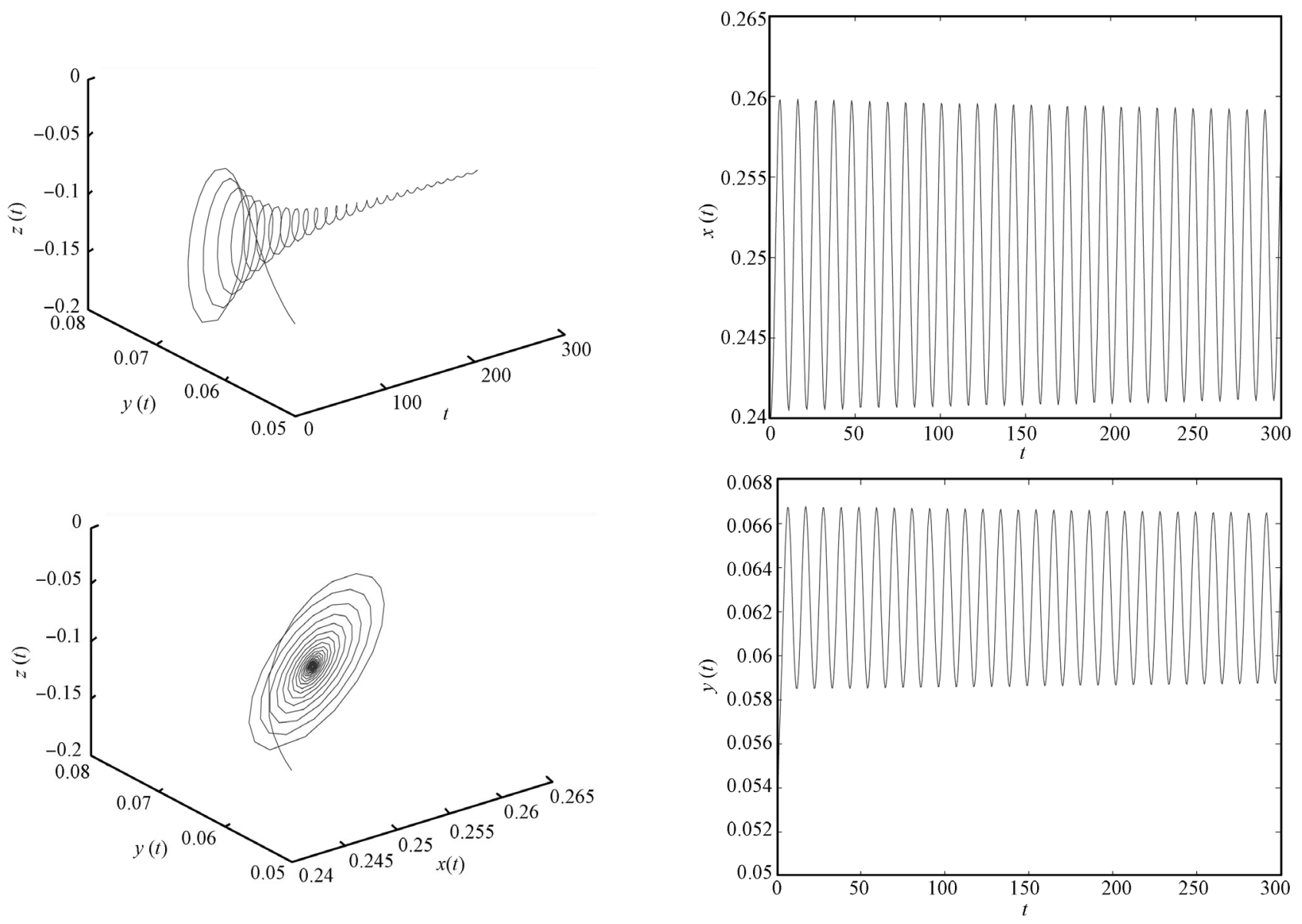

Fig. 2 Behavior and phase portraits of system (35) with $\alpha=$ $0.25<\alpha_{0} \approx 0.4512$. The equilibrium $\mathrm{E}\left(\frac{1}{4}, \frac{1}{16}, \frac{1}{4},-\frac{12}{125}\right)$ is asymptotically stable. The initial value is $(1.5,1.5,1.5)$

\section{Conclusions}

In this paper, a feedback control method is applied to suppress chaotic behavior of a Sprott E system within the chaotic attractor. By adding a continuous distributed time delayed force to the third equation of the Sprott E system, we have made a detailed discussion on the local stability of the equilibrium $\mathrm{E}\left(x^{*}, y^{*}, z^{*}\right)$ and local Hopf bifurcation of the delayed Sprott E system model. We showed that if some

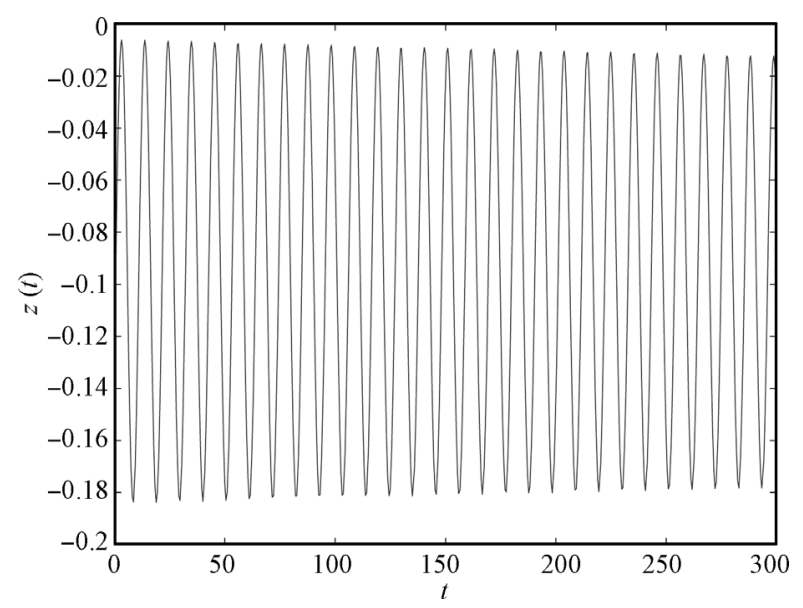



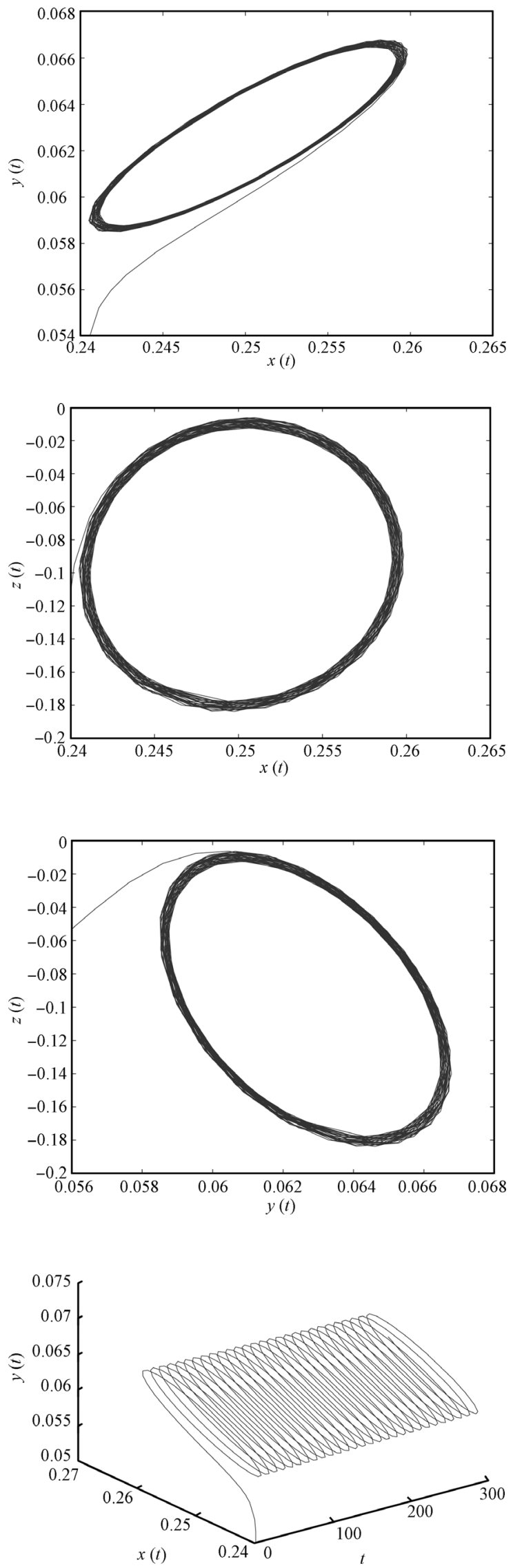
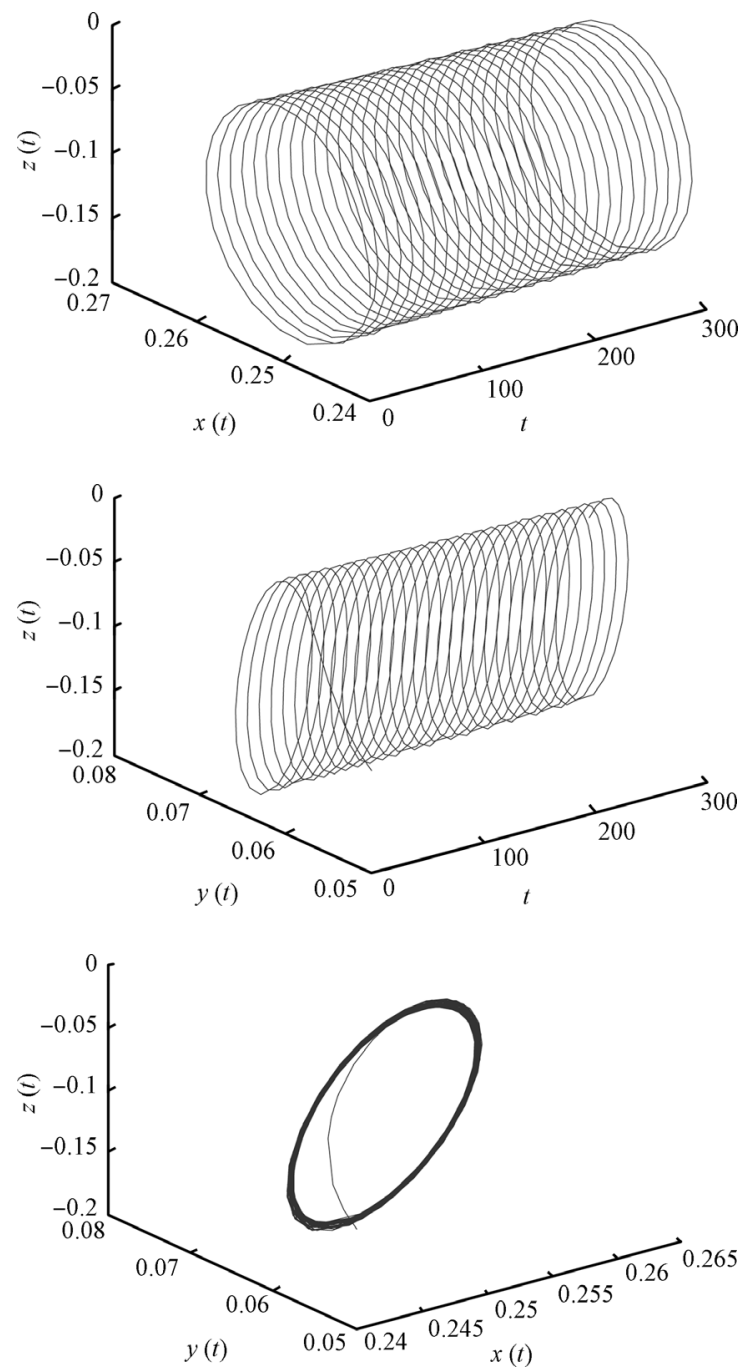

Fig. 3 Behavior and phase portraits of system (35) with $\alpha=$ $0.55>\alpha_{0} \approx 0.4512$. Hopf bifurcation occurs from the equilibrium $E\left(\frac{1}{4}, \frac{1}{16}, \frac{1}{4},-\frac{12}{125}\right)$. The initial value is $(1.5,1.5,1.5)$

\section{References}

[1] E. Ott, C. Grebogi, J. A. York. Controlling chaos. Physical Review Letters, vol. 64, no. 11, pp. 1196-1199, 1990.

[2] X. F. Liao, G. R. Chen, Hopf bifurcations and chaos analysis of chens system with distributed delays. Chaos, Solitons and Fractals, vol. 25, no. 1, pp. 197-220, 2005.

[3] G. Corso, F. B. Rizzato. Hamiltonian bifurcation leading to chaos in a low-energy relativistic wave-particle system. Physica D, vol. 80, no. 3, pp. 296-306, 1995.

[4] A. E. Gohary, A. S. Ruzaiza. Chaos and adaptive control in two prey, one predator system with nonlinear feedback. Chaos, Solitons and Fractals, vol.34, no. 2, pp. 443-453, 2007.

[5] Y. A. Kuznetsov, S. Rinaldi. Remarks on food chain dynamics. Mathematical Biosciences, vol. 134, no. 1, pp. 1-33, 1996.

[6] M. C. Varriale, A. A.Gomes. A study of a three species food chain. Ecological Modelling, vol.110, no. 2, pp.119133, 1998. 
[7] F. Y. Wang, G. P. Pang. Chaos and Hopf bifurcation of a hybrid ratio-dependent three species food chain. Chaos, Solitons \& Fractals, vol. 36, no. 5, pp. 1366-1376, 2008.

[8] J. G. Du, T. W. Huang, Z. H. Sheng, H. B. Zhang. A new method to control chaos in an economic system. Applied Mathematics and Computation, vol.217, no. 6, pp. 23702380, 2010.

[9] M. Sadeghpour, H. Salarieh, A. Alasty. Controlling chaos in tapping mode atomic force microscopes using improved minimum entropy control. Applied Mathematical Modelling, vol. 37, no. 3, pp. 1599-1606, 2013.

[10] A. S. Hegazi, E. Ahmed, A. E. Matouk. On chaos control and synchronization of the commensurate fractional order Liu system. Communications in Nonlinear Science and $\mathrm{Nu}$ merical Simulation, vol. 18, no. 5, pp. 1193-1202, 2013.

[11] G. R. Chen. Controlling Chaos and Bifurcations in Engineering Systems, Boca Raton, FL: CRC Press, 1999.

[12] X. S. Yang, G. R. Chen. Some observer-based criteria for discrete-time generalized chaos synchronization. Chaos, Solitons \& Fractals, vol. 13, no. 6, pp. 1303-1308, 2002.

[13] G. R. Chen, X. Dong. On feedback control of chaotic continuous-time systems. IEEE Transactions on Circuits and Systems, vol. 40, no. 9, pp. 591-601, 1993.

[14] M. T. Yassen. Chaos control of Chen chaotic dynamical system. Chaos, Solitons \& Fractals, vol. 15, no. 2, pp. 271283, 2003.

[15] H. N. Agiza. Controlling chaos for the dynamical system of coupled dynamos. Chaos, Solitons \& Fractals, vol. 13, no. 2, pp. 341-352, 2002.

[16] H. T. Zhao, Y. P. Lin, Y. X. Dai. Bifurcation analysis and control of chaos for a hybrid ratio-dependent three species food chain. Applied Mathematics and Computation, vol. 218, no. 5, pp. 1533-1546, 2011.

[17] Y. L. Song, J. J. Wei. Bifurcation analysis for Chen's system with delayed feedback and its application to control of chaos. Chaos, Solitons \& Fractals, vol. 22, no. 1, pp. 75-91, 2004.

[18] E. W. Bai, K. E. Lonngren. Sequential synchronization of two Lorenz systems using active control. Chaos, Solitons \& Fractals, vol. 11, no. 7, pp. 1041-1044, 2000.

[19] M. T. Yassen. Adaptive control and synchronization of a modified Chua's circuit system. Applied Mathematics and Computation, vol. 135, no. 1, pp. 113-128, 2001.

[20] T. L. Liao, S. H. Lin. Adaptive control and synchronization of Lorenz system. Journal of the Franklin Institute, vol. 336 , no. 6, pp. 925-937, 1999.

[21] H. Richter, K. J. Reinschke, H. Richter, K. J. Reinschke. Optimization of local control of chaos by an evolutionary algorithm. Physica D, vol. 144, no. 3-4, pp. 309-334, 2000.

[22] R. Senkerik, I. Zelinka, D. Davendra, Z. Oplatkova. Utilization of SOMA and differential evolution for robust stabilization of chaotic Logistic equation. Computers \& Mathematics with Applications, vol.60, no. 4, pp. 1026-1037, 2010.

[23] I. Zelinka, M. Chadli, D. Davendra, R. Senkerik, R. Jasek. An investigation on evolutionary reconstruction on continuous chaotic systems. Mathematical and Computer Modelling, vol. 57, no. 1-2, pp. 2-15, 2013.
[24] M. Chadli, I. Zelinka, T. Youssef. Unknown inputs observer design for fuzzy systems with application to chaotic system reconstruction. Computers and Mathematics with Applications, vol. 66, no. 2, pp. 147-154, 2013.

[25] H. A. Yousef, M. Hamdy. Observer-based adaptive fuzzy control for a class of nonlinear time-delay systems. International Journal of Automation and Computing, vol. 10, no. 4, pp. 275-280, 2013.

[26] G. D. Zhao, N. Duan. A continuous state feedback controller design for high-order nonlinear systems with polynomial growth nonlinearities. International Journal of $\mathrm{Au}$ tomation and Computing, vol. 10, no. 4, pp. 267-274, 2013.

[27] X. Wang, G. R. Chen. A chaotic system with only one stable equilibrium. Communications in Nonlinear Science and Numerical Simulation, vol. 17, no. 3, pp. 1264-1272, 2012.

[28] J. C. Sprott. Simplest dissipative chaotic flow. Physics Letters A, vol. 228, no. 3-4, pp. 271-274, 1997.

[29] X. Wang, G. R. Chen. Constructing a chaotic system with any number of equilibria. Nonlinear Dynamics, vol. 71, no. 3, pp. 429-436, 2013.

[30] B. Hassard, D. Kazarino, Y. Wan. Theory and Applications of Hopf bifurcation, Cambridge, UK: Cambridge University Press, 1981.

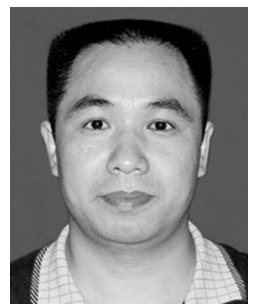

Chang-Jin Xu graduated from Huaihua University, China in 1994. He received His M. Sc. degree from Kunming University of Science and Technology, China in 2004 and his $\mathrm{Ph}$. D. degree from Central South University, China in 2010, respectively. He is currently a professor at the Guizhou Key Laboratory of Economics System Simulation in Guizhou University of Finance and Economics, China. He has published about 100 refereed journal papers. He is a reviewer of Mathematical Reviews, Zentralblatt MATH.

His research interests include stability and bifurcation theory of delayed differential equation.

E-mail: xcj403@126.com (Corresponding author)

ORCID iD: 0000-0001-5844-2985

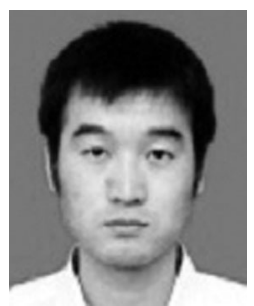

Yu-Sen Wu graduated from Liaocheng University, China in 2004. He received his M.Sc. degree from Central South University, China in 2007, and his Ph. D. degree from Central South University, China in 2010, respectively. He is currently an associate professor at School of Mathematics and Statistics of Henan University of Science and Technology. He has published about 30 refereed journal papers. He is a reviewer of Mathematical Reviews, Zentralblatt MATH.

His research interests include the qualitative theory of ordinary differential equation and computer symbol calculation.

E-mail: wuyusen82714@yahoo.com.cn 